\title{
Carreadores de oxigênio a base de Ni obtidos por diferentes métodos de síntese para aplicação no processo de reforma de metano com recirculação química
}

\section{(Ni-based oxygen carriers obtained by different synthesis methods for use in the chemical looping reforming of methane process)}

\author{
J. A. B. L. R. Alves ${ }^{1}$, G. P. Figueredo ${ }^{2,5}$, R. L. B. A. Medeiros ${ }^{3}$, T. R. Costa ${ }^{3}$, D. M. A. Melo ${ }^{2,3}$, M. A. F. Melo ${ }^{3,4}$ \\ ${ }^{1}$ Universidade Federal de Campina Grande - UFCG, Centro de Educação e Saúde, Campus Cuité, Cuité, PB, Brasil \\ ${ }^{2}$ Programa de Pós-Graduação em Química, \\ ${ }^{3}$ Programa de Pós-Graduação em Ciência e Engenharia de Materiais, \\ ${ }^{4}$ Programa de Pós-Graduação em Engenharia Química Universidade Federal do Rio Grande do Norte - UFRN, \\ Campus Universitário Lagoa Nova, CP 1524, Natal, RN, Brasil 59078-970 \\ ${ }^{5}$ Instituto Federal do Maranhão - IFMA, Departamento Acadêmico de Química, Av. Getúlio Vargas, 04, Monte \\ Castelo, São Luís, MA, Brasil 65030-005
}

Resumo

Carreadores de oxigênio a base de $\mathrm{Ni}$ e contendo $\mathrm{La}, \mathrm{Al}$ e $\mathrm{Mg}$ foram sintetizados com sucesso por combustão assistida por microondas (N6C), combustão assistida por microondas seguida de impregnação por via úmida (N6CI) e coprecipitação (N6CP), visando sua aplicação na produção de hidrogênio a partir de metano em sistemas de recirculação química com captura de $\mathrm{CO}_{2}$. Os materiais foram preparados obedecendo a fórmula geral $\mathrm{Ni} / \mathrm{La}_{2} \mathrm{O}_{3} / \mathrm{Al}_{2} \mathrm{O}_{3} / \mathrm{MgO}$, onde $\mathrm{x}=60 \%$ em massa, sendo o restante referente aos óxidos de lantânio (8\%), alumínio (30\%) e magnésio (2\%). Os sólidos obtidos foram caracterizados por difração de raios X, redução a temperatura programada e reatividade para o processo de reforma de metano com recirculação química. Os testes de reatividade foram conduzidos a $900{ }^{\circ} \mathrm{C}$, onde inicialmente a amostra foi submetida a 8 ciclos de CAO (capacidade de armazenamento de oxigênio), e finalmente foram realizados 2 ciclos de reação, com cada ciclo contendo 15 pulsos de metano e 15 pulsos de oxigênio. Os resultados das caracterizações indicam que os métodos empregados foram adequados à obtenção de diferentes materiais com propriedades distintas. De modo geral, os carreadores apresentaram excelentes percentuais de conversão de $\mathrm{CH}_{4}$ e elevada produção de hidrogênio. $\mathrm{O}$ carreador N6CP apresentou o melhor desempenho para produção de gás de síntese, com razão $\mathrm{H}_{2} / \mathrm{CO}$ igual a 2 no $15^{\circ}$ pulso, além de ser apontado como o mais resistente à desativação por coque e com maior potencial de oxidação, consumindo $100 \%$ de $\mathrm{O}_{2}$ até o $12^{\circ}$ pulso. Por sua vez, o N6CI perdeu bastante desempenho ao longo dos ciclos e o N6C formou a maior quantidade de coque, revelando as peculiaridades dos métodos de síntese empregados. De acordo com a distribuição dos produtos formados na etapa de redução com $\mathrm{CH}_{4}$, pode-se concluir que as reações mais frequentes foram craqueamento catalítico, combustão e reforma.

Palavras-chave: carreadores de oxigênio, combustão assistida por microondas, coprecipitação, reforma com recirculação química.

\section{Abstract}

Oxygen carriers containing Ni, La, Al and Mg were synthesized successfully by microwave assisted self-combustion (N6C), microwave assisted self-combustion followed by wet impregnation (N6CI) and co-precipitation (N6CP), for their application in the production of hydrogen from methane chemical recirculation systems with $\mathrm{CO}_{2}$ capture. The materials were prepared following the general formula $\mathrm{Ni} / \mathrm{La}_{2} \mathrm{O}_{3} / \mathrm{Al}_{2} \mathrm{O}_{3} / \mathrm{MgO}$, where $x=60 \%$ by weight, the remainder being related to lanthanum $(8 \%)$, aluminum (30\%) and magnesium (2\%) oxide. The obtained solids were characterized by X-ray diffraction), temperature programmed reduction and activity through methane reforming process with chemical recirculation. Reactivity tests were conducted at $900{ }^{\circ} \mathrm{C}$, where the sample was initially subjected to 8 cycles of OSC (oxygen storage capacity), and finally were carried out two reaction cycles, with each cycle containing 15 pulses of methane and 15 pulses of oxygen. The results of the characterizations indicate that the methods used were appropriate for obtaining different materials with different properties. Generally, the carriers showed excellent percentage of $\mathrm{CH}_{4}$ conversion, and high hydrogen production. The carrier N6CP exhibited the best performance to syngas production with $\mathrm{H}_{2} / \mathrm{CO}$ ratio equal to 2 on the $15^{\text {th }}$ pulse, as well as being appointed as being the most resistant to deactivation by coke and higher oxidation potential, consuming $100 \% \mathrm{O}_{2}$ up to the $12^{\text {th }}$ pulse. In turn, the N6CI lost enough performance throughout the cycles and the N6C formed larger amount of coke, revealing the peculiarities of the synthesis methods employed. According to the reaction products formed in the reduction step with $\mathrm{CH}_{4}$, it can be concluded that the most frequent reactions were catalytic cracking, reforming and combustion.

Keywords: oxygen carriers, microwave-assisted combustion, co-precipitation, chemical looping reforming. 


\section{INTRODUÇÃO}

$\mathrm{O}$ aumento da liberação de gás carbônico $\left(\mathrm{CO}_{2}\right)$ para atmosfera, proveniente de ações antropogênicas, tem despertado a atenção da comunidade científica. Considerando que o crescimento populacional é acompanhado pelo aumento da demanda energética, a liberação de $\mathrm{CO}_{2}$ se torna um problema grave. Segundo o Painel Intergovernamental sobre Mudanças Climáticas, a emissão de $\mathrm{CO}_{2}$ na atmosfera cresceu 39\% desde o período pré-industrial até o final de 2010 [1]. Portanto, torna-se necessário a busca de tecnologias que diminuam a liberação de $\mathrm{CO}_{2}$ para atmosfera sem afetar a produção de energia. A diminuição da emissão do $\mathrm{CO}_{2}$ por si só não resolve os problemas a ele atribuído. $\mathrm{A}$ utilização de gás hidrogênio $\left(\mathrm{H}_{2}\right)$ para geração de energia é uma solução promissora frente aos problemas ambientais causados pelos combustíveis fósseis, principalmente relacionados a liberação de $\mathrm{CO}_{2}$. Além de poder ser utilizado como combustível para o setor automobilístico, o $\mathrm{H}_{2}$ é muito utilizado na produção de metanol, amônia e na indústria petroquímica [2].

Hoje a principal forma para produzir $\mathrm{H}_{2}$ é a partir da reforma a vapor do metano. A geração do gás de síntese (CO $\mathrm{e}_{2}$ ) nesse processo exige que haja uma separação dos gases para a purificação do $\mathrm{H}_{2}$. Além disso, o calor necessário para a reforma é proveniente da queima de um combustível fora da coluna de reforma, o que gera muito $\mathrm{CO}_{2}$ e $\mathrm{N}_{2}$ misturado, necessitando de uma separação adicional por tecnologias de captura de carbono (CCS) [3]. Uma alternativa para a produção de gás hidrogênio a partir de um combustível fóssil com separação e captura do $\mathrm{CO}_{2}$ é a utilização do processo de CLR (chemical looping reforming) [3]. No processo de CLR são utilizados dois reatores, normalmente leitos fluidizados, conectados entre si, em que um é o reator de redução e o outro é o reator de oxidação. No reator de redução, um sólido na forma de óxido, chamado de carreador de oxigênio, reage com o combustível para formar o gás de síntese $\left(\mathrm{H}_{2} / \mathrm{CO}\right)$. $\mathrm{O}$ carreador de oxigênio na forma reduzida passa para o reator de oxidação para ser oxidado e dar início a um novo ciclo. Esse processo utiliza os mesmos conceitos do processo de CLC (chemical looping combustion), porém o produto desejado não é $\mathrm{H}_{2} \mathrm{O}$ e $\mathrm{CO}_{2}$, mas o gás de síntese $\left(\mathrm{H}_{2} / \mathrm{CO}\right)$. A diferença está basicamente na alimentação do combustível, em que a razão ar/combustível deve ser mantida baixa para não favorecer a oxidação do $\mathrm{H}_{2}$ e $\mathrm{CO}$ a $\mathrm{H}_{2} \mathrm{O}$ e $\mathrm{CO}_{2}$, respectivamente [4].

A seleção correta do carreador de oxigênio é a chave para o bom funcionamento do sistema CLR. Neste processo, a reforma é uma importante reação que produz a mistura $\mathrm{H}_{2} / \mathrm{CO}$ no reator de combustível, e é de se esperar que o carreador de oxigênio seja ativo na reação de reforma convencional [4]. Os catalisadores utilizados nos processos de reforma geralmente usam o Ni como metal ativo suportado em $\mathrm{Al}_{2} \mathrm{O}_{3}[5,6]$. A adição de pequenas quantidades de óxidos de $\mathrm{Mg}, \mathrm{Ca}$, $\mathrm{La}$ e Co aumentou a estabilidade química dos carreadores, impedindo sua desativação ao longo de vários ciclos de oxidação/redução $[7,8]$, o que os tornam importantes e vantajosos para estudos de aplicação em CLR. Há relatos [9] de que o acréscimo de La no carreador Ni$\mathrm{Al}_{2} \mathrm{O}_{3}$ aumenta a estabilidade da partícula de níquel frente a oxidação/redução durante vários ciclos. A adição de metais alcalinos terrosos como $\mathrm{Mg}$ e $\mathrm{Ca}$ diminuem a formação de coque, principalmente quando o metal ativo é o Ni. A presença de $\mathrm{Co}$ em $\mathrm{Ni}-\mathrm{Al}_{2} \mathrm{O}_{3}$ aumentou a estabilidade do suporte durante a etapa de redução com $\mathrm{CH}_{4}$ em processo CLR, diminuindo a interação entre o níquel e o suporte, evitando a formação do espinélio $\mathrm{NiAl}_{2} \mathrm{O}_{4}[10]$. $\mathrm{O} \mathrm{NiAl}_{2} \mathrm{O}_{4}$ diminui a taxa reacional durante a redução do combustível no reator de combustão [11]. Esse óxido é um espinélio de estrutura $\mathrm{AB}_{2} \mathrm{O}_{4}$ apresentando os íons $\mathrm{Ni}^{2+} \mathrm{e} \mathrm{Al}^{3+}$ nos sítios $\mathrm{A}$ e $\mathrm{B}$ da estrutura, respectivamente $[12,13]$. Portanto, o presente trabalho teve como objetivo sintetizar carreadores de oxigênio à base de níquel e contendo $\mathrm{La}, \mathrm{Al}$ e $\mathrm{Mg}$, por três métodos diferentes, e estudar a distribuição dos produtos formados durante a reação com $\mathrm{CH}_{4}$ em ciclos. Diferentes caminhos de síntese são desejáveis já que geram materiais com diferentes propriedades estruturais e texturais, proporcionando novos materiais com distintas propriedades como carreadores de oxigênio.

\section{EXPERIMENTAL}

\section{Sintese dos carreadores de oxigênio}

A síntese dos carreadores de oxigênio foi realizada através dos métodos de combustão assistida por microondas [14-16], seguida de impregnação por via úmida [17] e coprecipitação $[18,19]$, com as devidas adaptações. Os carreadores apresentam composição química $\mathrm{Ni}_{x} / \mathrm{La}_{2} \mathrm{O}_{3} /$ $\mathrm{Al}_{2} \mathrm{O}_{3} / \mathrm{MgO}$, em que $\mathrm{x}=60 \%$ em massa, sendo o restante referente aos óxidos de lantânio (8\%), alumínio (30\%) e magnésio (2\%). No método de combustão assistida por microondas, as quantidades necessárias de cada nitrato de $\mathrm{Ni}, \mathrm{La}, \mathrm{Al}$ e $\mathrm{Mg}$ foram misturadas com $10 \mathrm{~mL}$ de água deionizada em um béquer. Após homogeneização e completa solubilização dos sais, foi adicionada uréia (2 moles de nitrato/5 moles de uréia) como combustível. Em seguida, o béquer contendo a mistura foi colocado em um forno de microondas convencional a uma potência de 650 W e frequência de 2,45 GHz até a autoignição ocorrer (4-6 min). A amostra foi nomeada como N6C.

No método de combustão assistida por microondas seguida de impregnação por via úmida, os nitratos de $\mathrm{La}, \mathrm{Al}$ e Mg foram misturados com $10 \mathrm{~mL}$ de água desionizada em um béquer. Após homogeneização e completa solubilização dos sais, foi adicionada uréia ( 2 moles de nitrato/ 5 moles de uréia) como combustível. Em seguida, o béquer contendo a mistura foi colocado em um forno de microondas convencional a uma potência de $650 \mathrm{~W}$ e frequência de 2,45 $\mathrm{GHz}$ até a autoignição ocorrer (4 - 6 min). $\mathrm{O}$ material obtido nessa etapa foi nomeado de LAM. A amostra do material obtido anteriormente (LAM) foi impregnada com $60 \%$ de níquel, usando o precursor nitrato de níquel hexahidratado dissolvido em $10 \mathrm{~mL}$ de 2-propanol, através do processo de 
contato [17]. O suporte (LAM), juntamente com o nitrato de níquel dissolvido no álcool, foi submetido à agitação lenta por $2 \mathrm{~h}$ a $55^{\circ} \mathrm{C}$ até completa evaporação do álcool, e então calcinados a $850^{\circ} \mathrm{C}$ por $4 \mathrm{~h}$. Essa amostra foi nomeada como N6CI. No método de coprecipitação, os nitratos metálicos foram precipitados por gotejamento da solução aquosa de $\mathrm{NH}_{4} \mathrm{OH}(1 \mathrm{M})$ a $55^{\circ} \mathrm{C}$ e sob agitação até a formação de um precipitado gelatinoso. $\mathrm{O}$ material foi então filtrado, lavado e seco em estufa a $110{ }^{\circ} \mathrm{C}$ por $12 \mathrm{~h}$. O sólido resultante foi calcinado a $850{ }^{\circ} \mathrm{C}$ por $4 \mathrm{~h}$. Essa amostra foi nomeada como N6CP.

\section{Caracterização dos carreadores de oxigênio}

Os pós sintetizados (N6C, LAM, N6CI e N6CP) tiveram as fases cristalinas identificadas por difração de raios $\mathrm{X}$ (DRX) em um difratômetro de raios X da Shimadzu, XRD7000 , que opera com radiação $\mathrm{Cu}-\mathrm{K} \alpha(\lambda=1,542 \AA)$ a 30 $\mathrm{kV}$ e $30 \mathrm{~mA}$. Os difratogramas foram obtidos na faixa $2 \theta$ de 10 a $80^{\circ}$ em passos de $0,02^{\circ}$ e tempo de passo de $1 \mathrm{~s}$ a temperatura ambiente de $25{ }^{\circ} \mathrm{C}$. A aquisição e o tratamento dos difratogramas foram realizados no software X'pert HighScore Plus da PANalytical. A posição e intensidade dos picos observados foram comparadas com dados fornecidos pelos arquivos de referência JCPDS (Joint Commitee on Powder Diffraction Standards) contidas na base de dados do ICDD (International Centre for Diffraction Data) versão 2003.

Os testes de redução a temperatura programada foram realizados em um equipamento da Quantacrome 2000. Para os testes, uma massa de cerca de $50 \mathrm{mg}$ de amostra foi colocada em um tubo de quartzo em forma de $U(6,6 \mathrm{~mm}$ de diâmetro interno) e submetida a um fluxo de $20 \mathrm{~mL} \cdot \mathrm{min}^{-1}$ da mistura redutora, que consistiu em $10 \%$ de hidrogênio diluído em nitrogênio. A temperatura da amostra foi então aumentada desde a temperatura ambiente até $900{ }^{\circ} \mathrm{C}$ a uma taxa de $10{ }^{\circ} \mathrm{C} \cdot \mathrm{min}^{-1}$.

Teste de reatividade: o teste de reatividade dos carreadores com foco no processo de reforma com recirculação química foi realizado em um equipamento Autochem II da Micromeritics com um espectrômetro de massas HPR20 acoplado, Hiden, onde a programação de pulsos (30 s) dos reagentes é preparada no sistema Autochem II e os gases de saída são analisados pelo espectrômetro de massas. Cada pulso consiste em um volume de $15,89 \mu \mathrm{L}$ para cada gás. $\mathrm{Na}$ análise, $100 \mathrm{mg}$ do carreador de oxigênio foi colocado no reator do sistema da Autochem II e sua temperatura de reação foi elevada até $900{ }^{\circ} \mathrm{C}$ sob vazão de $50 \mathrm{~mL} \cdot \mathrm{min}^{-1}$ de um gás inerte $(\mathrm{He})$. Após atingir essa temperatura, foram realizados 10 pulsos de $\mathrm{N}_{2}$ para realizar a respectiva correção da calibração. Em seguida, a amostra foi submetida a 8 ciclos de CAO (capacidade de armazenamento de oxigênio - do inglês Oxygen Storage Capacity - OSC). Cada ciclo é composto por 40 pulsos do agente redutor $\left(\mathrm{H}_{2}\right)$ seguido de 20 pulsos do agente oxidante $\left(\mathrm{O}_{2}\right)$. Após a realização do CAO, foram realizados 2 ciclos de reação, com cada ciclo contendo 15 pulsos de metano (etapa de redução) e 15 pulsos de oxigênio (etapa de oxidação). A quantificação de água nos produtos foi realizada por balanço de massa.

\section{RESULTADOS E DISCUSSÃO}

\section{Análise por difração de raios $X(D R X)$}

Os difratogramas de raios $\mathrm{X}$ para as amostras dos carreadores de oxigênio sintetizados pelos três métodos são apresentados na Fig. 1. É possível observar a formação majoritária da fase $\mathrm{LaAlO}_{3}$ (JCPDS n ${ }^{\circ}$ 082-0478) em todas as amostras (N6C, LAM, N6CI e N6CP). O $\mathrm{LaAlO}_{3}$ obtido por combustão assistida por microondas usando uréia como combustível já foi reportado na literatura [20], onde se observou a formação desse material sem a realização de tratamento térmico adicional. Ressalta-se que a formação do $\mathrm{LaAlO}_{3}$ em todos os carreadores sintetizados para esse estudo foi importante devido a sua alta estabilidade química e física sob atmosferas redutoras e oxidantes [21]. Para a amostra N6C (Fig. 1a), a identificação dos picos de difração mostrou o padrão de difração característico da fase $\mathrm{La}\left(\mathrm{Ni}_{0,5} \mathrm{Al}_{0,5}\right) \mathrm{O}_{3,05}$ (JCPDS $\mathrm{n}^{\circ}$ 083-0757). A substituição parcial do Al pelo Ni no sítio $\mathrm{B}$ da perovskita pseudocúbica desloca o pico próximo a $33,45^{\circ}(2 \theta)$, que está relacionado ao plano (110) do $\mathrm{LaAlO}_{3}$, para valores mais baixos, em torno de $33,10^{\circ}(2 \theta)$, que está relacionado ao plano (110) do $\mathrm{La}\left(\mathrm{Ni}_{0,5} \mathrm{Al}_{0,5} \mathrm{O}_{3,05}\right.$ [22].

A temperatura reacional durante a síntese por combustão provoca a interação do $\mathrm{NiO}$ com $\mathrm{o} \mathrm{Al}_{2} \mathrm{O}_{3}$, formando o espinélio $\mathrm{NiAl}_{2} \mathrm{O}_{4}$ (JCPDS $\mathrm{n}^{\circ}$ 073-0239). A literatura afirma que o espinélio $\mathrm{NiAl}_{2} \mathrm{O}_{4}$ atua melhor como suporte para o $\mathrm{NiO}$ do que como carreador de oxigênio, pois a redutibilidade desta fase é dificultada [23]. Identificou-se também a formação de $\mathrm{NiO}$ cúbico livre (JCPDS n ${ }^{\circ} 073-$ 1523) que corresponde, essencialmente, como o carreador de oxigênio. Não houve identificação dos picos referentes ao $\mathrm{MgO}$ devido à sua alta dispersão por toda a superfície do material ou por ter ficado localizado na parte amorfa do

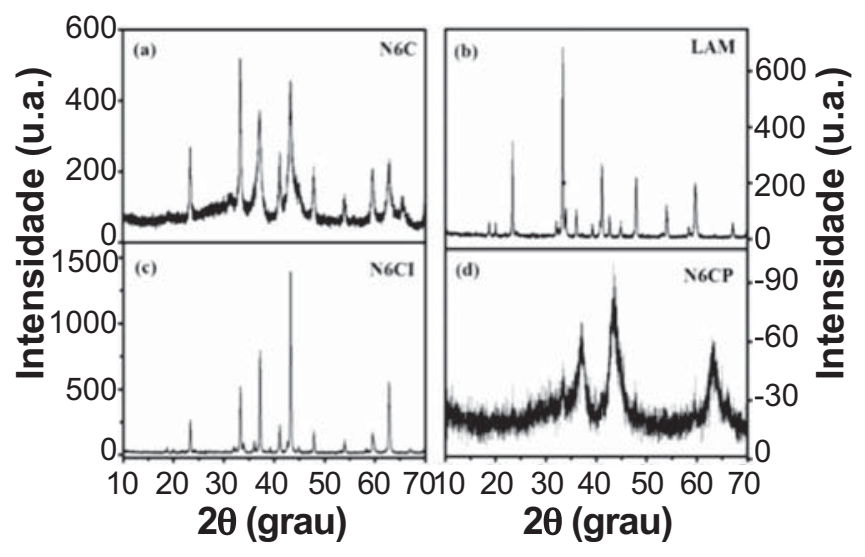

Figura 1: Difratogramas de raios X para as amostras (a) N6C, (b) LAM, (c) N6CI e (d) N6CP.

[Figure 1: X-ray diffraction patterns of samples (a) N6C, (b) LAM, (c) N6CI, and (d) N6CP.] 
material. Essas duas fases foram identificadas também para a amostra N6CP (Fig. 1d). Para o suporte LAM (Fig. 1b), identificou-se a formação de $\mathrm{MgO}\left(\mathrm{La}_{0,995} \mathrm{Al}_{1,05} \mathrm{O}_{3}\right)\left(\mathrm{Al}_{2} \mathrm{O}_{3}\right)_{5} \mathrm{e}$ $\mathrm{LaAlO}_{3}$. Por sua vez, na amostra N6CI (Fig. 1c), impregnada com Ni, houve a identificação das mesmas fases do suporte LAM e a formação de $\mathrm{NiO}$ cúbico livre (JCPDS $\mathrm{n}^{\circ}$ 0731523), com os planos de difração localizados em $2 \theta=37,25^{\circ}$, $43,27^{\circ}, 62,85^{\circ}, 75,41^{\circ}$ e $79,38^{\circ}$ e com os respectivos índices de Miller (111), (200), (220), (311) e (222). Isso mostra que não há interação significativa entre o $\mathrm{NiO}$ e o suporte. Por fim, pode-se observar na Fig. 1d que o difratograma da amostra N6CP apresenta muito ruído, mostrando que parte do material é amorfo. Entretanto, foi observado a formação das fases $\mathrm{LaAlO}_{3}\left(\mathrm{JCPDS} \mathrm{n}^{\circ}\right.$ 082-0478) e o espinélio $\mathrm{NiAl}_{2} \mathrm{O}_{4}$ (JCPDS nं 073-0239).

\section{Redução à temperatura programada (RTP)}

A Fig. 2 mostra os perfis de redução à temperatura programada para os carreadores de oxigênio sintetizados pelos três métodos. Pode-se observar que o suporte inerte (LAM), preparado apenas com $\mathrm{La}, \mathrm{Al}$ e $\mathrm{Mg}$, não apresenta eventos de redução durante toda a faixa de temperatura. Por sua vez, na amostra N6C são observados três eventos de redução. O primeiro ocorre de 395 a $450{ }^{\circ} \mathrm{C}$ e corresponde à redução do $\mathrm{NiO}$ livre a $\mathrm{Ni}^{0}$ [24]. O segundo na faixa de 600 ${ }^{\circ} \mathrm{C}$ representa a redução do $\mathrm{NiO}$ com maior interação com a fase $\mathrm{LaAlO}_{3}$ e o terceiro evento, em $788{ }^{\circ} \mathrm{C}$, é atribuído à fase $\mathrm{NiAl}_{2} \mathrm{O}_{4}[25,26]$, sendo o pico de maior intensidade. Este composto, por apresentar estabilidade térmica maior, necessita de uma maior quantidade de energia para que ocorra a redução [27].

Para a amostra N6CI, há dois picos de redução, com os máximos em 321 e $427{ }^{\circ} \mathrm{C}$. Na temperatura mais baixa $\left(321{ }^{\circ} \mathrm{C}\right.$ ) ocorre a redução de $\mathrm{NiO}$ a $\mathrm{Ni}^{0}$. O pico com o máximo em $427{ }^{\circ} \mathrm{C}$ também pode estar relacionado com

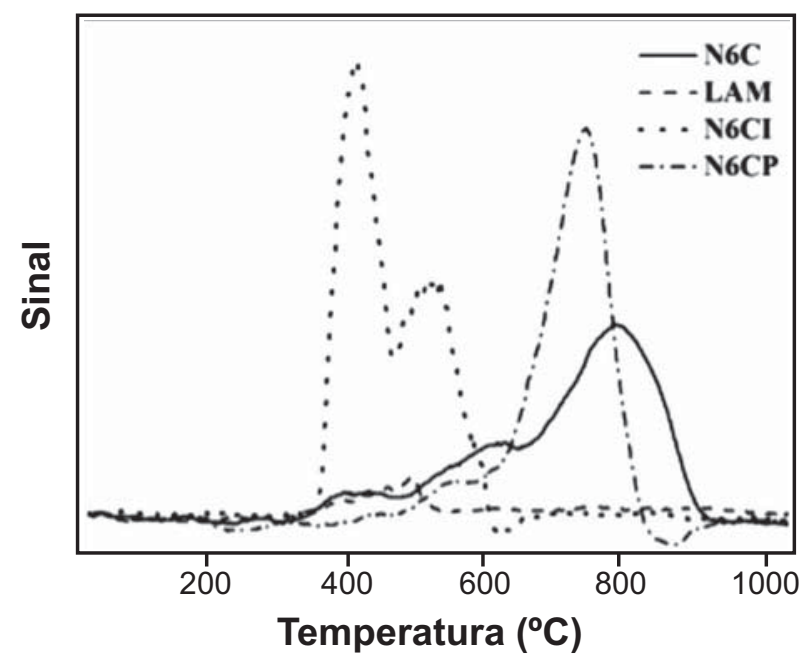

Figura 2: Perfis de redução à temperatura programada (RTP) para as amostras N6C, LAM, N6CI e N6CP.

[Figure 2: Temperature-programmed reduction (TPR) profiles of the samples N6C, LAM, N6CI and N6CP.] a redução do $\mathrm{NiO}$ a $\mathrm{Ni}^{0}$, pois não foi verificado pelo $\mathrm{DRX}$ nenhuma fase que corresponda à interação do $\mathrm{Ni}$ com o suporte ou com outro óxido presente na composição química utilizada. Para a amostra N6CP, observam-se dois picos de redução, sendo o primeiro pico em torno de 440 ${ }^{\circ} \mathrm{C}$ e o segundo em torno de $620^{\circ} \mathrm{C}$. O pico em $440{ }^{\circ} \mathrm{C}$ está relacionado à redução do $\mathrm{NiO}$ livre $\mathrm{a} \mathrm{Ni}{ }^{0}$. Por sua vez, o pico em torno de $620^{\circ} \mathrm{C}$ está relacionado à redução de $\mathrm{Ni}_{\mathrm{x}} \mathrm{Al}_{2} \mathrm{O}_{3+\mathrm{x}}$, que é um aluminato de níquel não-estequiométrico amorfo ou pode ser atribuído a espécies de $\mathrm{NiO}$ que interagem fracamente com o suporte. A literatura [23] mostra que para o $\mathrm{NiAl}_{2} \mathrm{O}_{4}$ cristalino o pico de redução, nas mesmas condições ou em condições parecidas de análise, sempre aparece em temperaturas superiores a $700{ }^{\circ} \mathrm{C}$, devido à estabilidade estrutural do $\mathrm{NiAl}_{2} \mathrm{O}_{4}$.

\section{Testes de reatividade - Estudo da etapa de redução}

A Fig. 3 mostra os percentuais de conversão de $\mathrm{CH}_{4}$ para os materiais estudados. Pode-se verificar que todos os carreadores apresentaram altas conversões de $\mathrm{CH}_{4}$. Para os carreadores $\mathrm{N} 6 \mathrm{C}$ e $\mathrm{N} 6 \mathrm{CP}$, as conversões foram semelhantes e apenas no último pulso há um decréscimo de conversão, relacionado à desativação dos carreadores. Para o material N6CI, ocorreu a desativação gradual a partir do $9^{\circ}$ pulso. A Fig. 4 mostra a distribuição dos produtos provenientes da conversão de $\mathrm{CH}_{4}$ durante a etapa de redução ao longo dos 15 pulsos para os carreadores de oxigênio.

A Fig. 4 mostra que os carreadores N6CI e N6CP tiveram comportamentos semelhantes ao longo dos pulsos na etapa de redução. Através dos gráficos (a) e (d) da Fig. 4, pode-se observar que houve a formação de $\mathrm{CO}_{2}$ e $\mathrm{H}_{2} \mathrm{O}$ nos instantes iniciais da etapa de redução com $\mathrm{CH}_{4}$ para os carreadores $\mathrm{N} 6 \mathrm{CI}$ e $\mathrm{N} 6 \mathrm{CP}$, havendo uma diminuição da formação de $\mathrm{CO}_{2}$ somente a partir do $7^{\circ}$ pulso. As reações envolvidas são claramente de combustão do metano, estando de acordo com a Equação A.

$$
\mathrm{CH}_{4(\mathrm{~g})}+4 \mathrm{NiO}_{(\mathrm{s})} \rightarrow 4 \mathrm{Ni}_{(\mathrm{s})}+\mathrm{CO}_{2(\mathrm{~g})}+2 \mathrm{H}_{2} \mathrm{O}_{(\mathrm{g})}
$$

A partir do $11^{\circ}$ pulso não foi identificada a formação de $\mathrm{CO}_{2}$. A diminuição da formação de $\mathrm{CO}_{2}$ é acompanhada pela formação de $\mathrm{CO}$ (Fig. 4 b). À medida que a reação de combustão vai ocorrendo, as partículas de níquel vão sendo reduzidas $\mathrm{a} \mathrm{Ni}^{0}$, e então uma reação de reforma do metano com o próprio $\mathrm{CO}_{2}$ ou $\mathrm{H}_{2} \mathrm{O}$ produzido ocorre, formando o $\mathrm{CO}$, segundo as Equações B, C e D.

$$
\begin{aligned}
& \mathrm{CH}_{4(\mathrm{~g})}+\mathrm{CO}_{2(\mathrm{~g})} \leftrightarrow 2 \mathrm{CO}_{(\mathrm{g})}+2 \mathrm{H}_{2(\mathrm{~g})} \\
& \mathrm{CH}_{4(\mathrm{~g})}+\mathrm{H}_{2} \mathrm{O}_{(\mathrm{g})} \leftrightarrow \mathrm{CO}_{(\mathrm{g})}+3 \mathrm{H}_{2(\mathrm{~g})} \\
& \mathrm{CH}_{4(\mathrm{~g})}+\mathrm{NiO}_{(\mathrm{s})} \rightarrow \mathrm{Ni}_{(\mathrm{s})}+\mathrm{CO}_{(\mathrm{g})}+2 \mathrm{H}_{2(\mathrm{~g})}
\end{aligned}
$$

A formação de $\mathrm{H}_{2} \mathrm{O}$ (Fig. 4d) mostra que todos os carreadores formaram $\mathrm{H}_{2} \mathrm{O}$ logo nos instantes iniciais da etapa de redução com o metano, estabilizando até o $7^{\circ}$ pulso. A partir daí, a reação de reforma com o próprio $\mathrm{CO}_{2}$ ou $\mathrm{H}_{2} \mathrm{O}$ 
produzido se inicia, formando o $\mathrm{H}_{2}$. Para o carreador N6C, há formação de $\mathrm{H}_{2} \mathrm{O}$ até o $7^{\circ}$ pulso, assim como todos os outros carreadores. Porém, identificou-se apenas uma discreta formação de $\mathrm{CO}_{2}$ e $\mathrm{CO}$ até o $5^{\circ}$ e $7^{\circ}$ pulso, respectivamente. $\mathrm{O}$ baixo teor de $\mathrm{CO}_{2}$ formado é uma indicação de que a temperatura do teste não foi suficiente para proporcionar a redução do níquel da estrutura do óxido formado na síntese direta de combustão por microondas. Além disso, pode ter ocorrido o craqueamento catalítico do $\mathrm{CH}_{4}$, que produz $\mathrm{C}+$ $2 \mathrm{H}_{2}$ (Equação E), onde a maior parte do $\mathrm{H}_{2}$ produzido pode reagir com $\mathrm{NiO}$ existente na superfície do carreador, sendo então oxidado a $\mathrm{H}_{2} \mathrm{O}$, como está representado na Equação $\mathrm{F}$.

$$
\begin{aligned}
& \mathrm{CH}_{4(\mathrm{~g})} \rightarrow \mathrm{C}_{(\mathrm{s})}+2 \mathrm{H}_{2(\mathrm{~g})} \\
& \mathrm{H}_{2(\mathrm{~g})}+\mathrm{NiO}_{(\mathrm{s})} \rightarrow 4 \mathrm{Ni}_{(\mathrm{s})}+\mathrm{H}_{2} \mathrm{O}_{(\mathrm{g})}
\end{aligned}
$$

Do $7^{\circ}$ ao $11^{\circ}$ pulso, há a formação de $\mathrm{CO}_{2}, \mathrm{H}_{2} \mathrm{O}, \mathrm{CO}$ e $\mathrm{H}_{2}$. Porém, a quantidade de $\mathrm{H}_{2} \mathrm{O}$ formada vai diminuindo (Fig. 4d) e a de $\mathrm{H}_{2}$ vai aumentando (Fig. 4c) ao longo dessa faixa, o que pode estar relacionado às reações paralelas que consomem $\mathrm{H}_{2} \mathrm{O}$ e produzem $\mathrm{H}_{2}$, como mostrado nas Equações $\mathrm{G}$ e H, sendo a reação representada pela Equação $\mathrm{J}$ mais pronunciada.
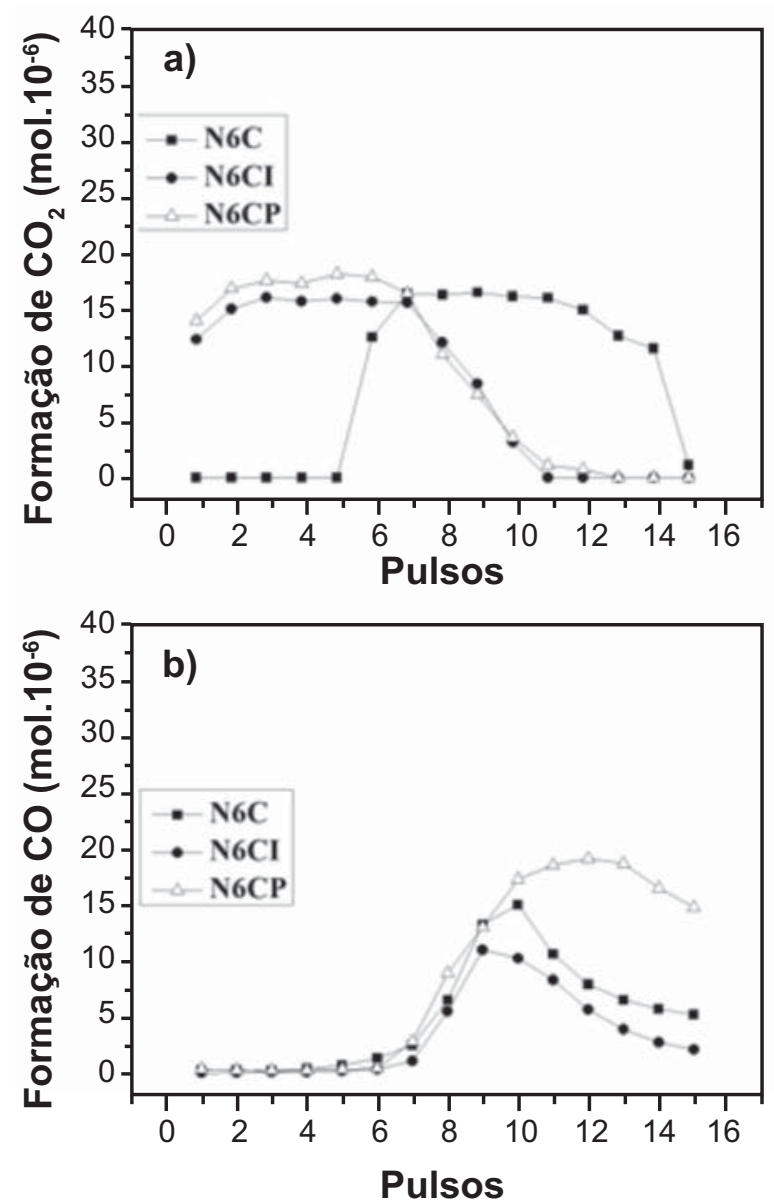

$$
\begin{aligned}
& \mathrm{C}_{(\mathrm{s})}+\mathrm{H}_{2} \mathrm{O}_{(\mathrm{g})} \leftrightarrow \mathrm{CO}_{(\mathrm{g})}+\mathrm{H}_{2(\mathrm{~g})} \\
& \mathrm{CO}_{(\mathrm{g})}+\mathrm{H}_{2} \mathrm{O}_{(\mathrm{g})} \leftrightarrow \mathrm{CO}_{2(\mathrm{~g})}+\mathrm{H}_{2(\mathrm{~g})}
\end{aligned}
$$

No entanto, a reação reversa de deslocamento de água (Equação I) também ocorre, contrabalanceando as quantidades dos produtos formados.

$$
\mathrm{CO}_{2(\mathrm{~g})}+\mathrm{H}_{2(\mathrm{~g})} \leftrightarrow \mathrm{CO}_{(\mathrm{g})}+\mathrm{H}_{2} \mathrm{O}_{(\mathrm{g})}
$$

Como não houve a desativação do carreador N6C até o $11^{\circ}$ pulso, supõe-se que o carbono formado pela reação de craqueamento do metano está sendo continuamente removido. A remoção do carbono formado pelo craqueamento do metano pode ser realizada pelo oxigênio proveniente do suporte ou pela dissociação do $\mathrm{CO}_{2}$. De acordo com [28], o oxigênio do suporte oxida o carbono e a reação de dissociação do $\mathrm{CO}_{2}$ reabastece o oxigênio do suporte, segundo as Equações J e K.

$$
\begin{aligned}
& \mathrm{C}_{(\mathrm{s})}+\mathrm{O}_{\text {ads. }} \rightarrow \mathrm{CO} \\
& \mathrm{CO}_{2(\mathrm{~g})} \rightarrow \mathrm{CO}_{(\mathrm{g})}+\mathrm{O}_{\text {ads. }}
\end{aligned}
$$
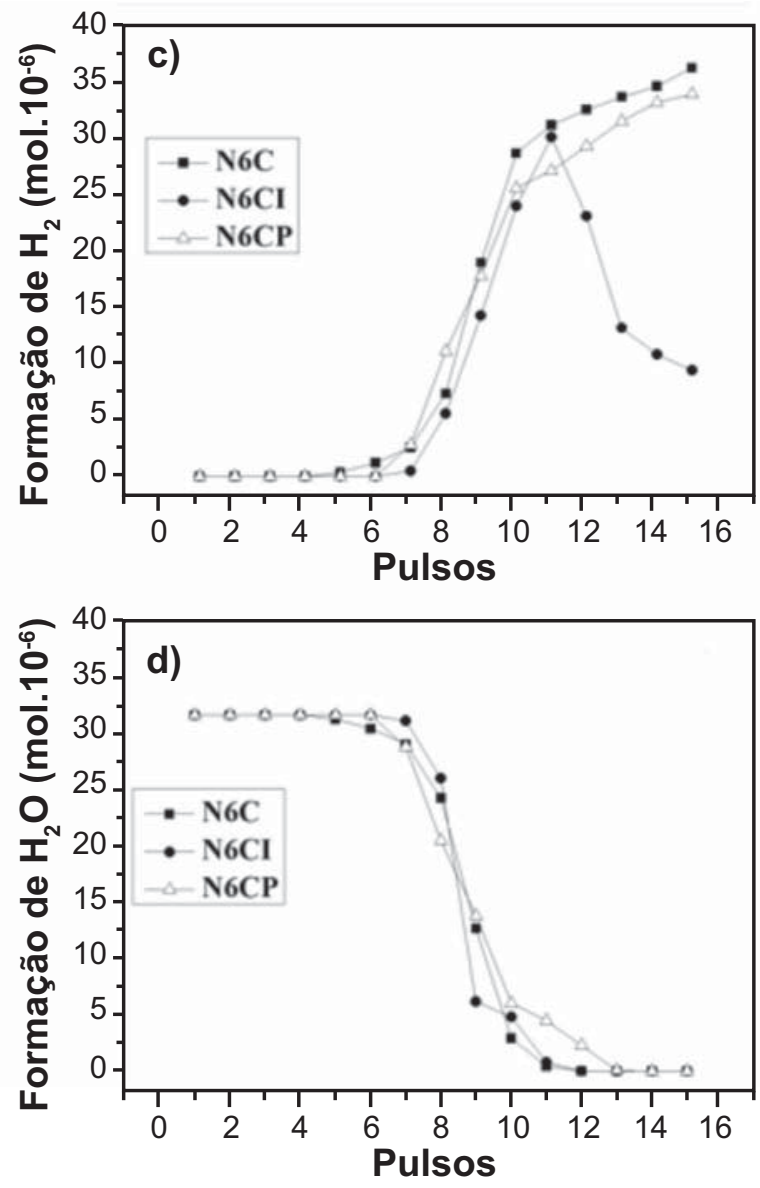

Figura 4: Distribuição dos produtos na etapa de redução para os carreadores de oxigênio. Formação de (a) $\mathrm{CO}_{2}$, (b) $\mathrm{CO}$, (c) $\mathrm{H}_{2}$ e (d) $\mathrm{H}_{2} \mathrm{O}$. [Figure 4: Distribution of products in the reduction step for oxygen carriers. Formation of (a) $\mathrm{CO}_{2}$, (b) $\mathrm{CO}$, (c) $\mathrm{H}_{2}$, and (d) $\mathrm{H}_{2} \mathrm{O}$.] 


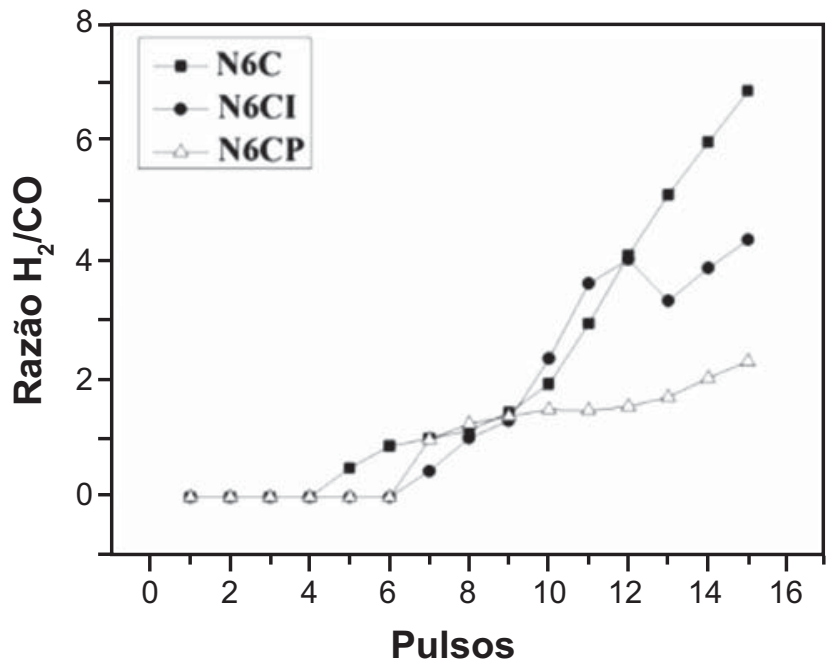

Figura 5: Razão $\mathrm{H}_{2} / \mathrm{CO}$ para os carreadores de oxigênio. [Figure 5: $\mathrm{H}_{2} / \mathrm{CO}$ ratio for the oxygen carrier.]

Do $12^{\circ}$ ao $14^{\circ}$ pulso, há a formação de $\mathrm{H}_{2}, \mathrm{CO}$ e $\mathrm{CO}_{2}$, que também podem ser explicados pelas equações químicas supracitadas. No $15^{\circ}$ pulso, ocorre uma diminuição drástica do consumo de $\mathrm{CH}_{4}$ provavelmente devido à deposição de coque, segundo a Equação E. Observa-se uma tendência global em relação à produção de hidrogênio para os materiais investigados da seguinte forma: N6C > N6CP > N6CI.

A Fig. 5 mostra a razão $\mathrm{H}_{2} / \mathrm{CO}$ para os carreadores de oxigênio. $\mathrm{O}$ gráfico mostra que a quantidade de $\mathrm{H}_{2}$ produzida foi elevada para os três materiais estudados e cresce continuamente ao longo dos pulsos, corroborando com as discussões anteriores, atingindo o máximo no último pulso. Tomando-se o último pulso como exemplo, a razão $\mathrm{H}_{2} / \mathrm{CO}$ para o N6CP é igual a 2, que pode estar relacionado a uma reação de oxidação parcial do metano (Equação L). A razão $\mathrm{H}_{2} / \mathrm{CO}$ para o N6CI e N6C é igual a 4 e 7, respectivamente. A alta quantidade de $\mathrm{H}_{2}$ produzido em relação ao $\mathrm{CO}$ é consistente com a reação de craqueamento do metano (Equação E). Portanto, o carreador preparado por coprecipitação (N6CP) aponta como sendo o mais resistente à desativação por coque do que os demais carreadores (N6C e N6CI). De acordo com a distribuição dos produtos formados na etapa de redução com $\mathrm{CH}_{4}$, observa-se uma tendência global com relação às reações envolvidas: craqueamento catalítico $>$ combustão $>$ reforma.

$$
\mathrm{CH}_{4(\mathrm{~g})}+1 / 2 \mathrm{O}_{2(\mathrm{~g})} \rightarrow 2 \mathrm{H}_{2(\mathrm{~g})}+\mathrm{CO}_{(\mathrm{g})}
$$

\section{Estudo da etapa de oxidação}

Após os pulsos utilizando o $\mathrm{CH}_{4}$ como redutor na etapa de redução, há a oxidação dos carreadores empregando-se $\mathrm{O}_{2}$ como oxidante. A Fig. 6 mostra os percentuais de conversão de $\mathrm{O}_{2}$ ao longo dos 15 ciclos de teste. Até o $8^{\circ}$ pulso, todos os carreadores consomem $100 \%$ do $\mathrm{O}_{2}$. Após esse pulso, os carreadores N6C e N6CI começam a consumir menos oxigênio, e o carreador N6CP continua a consumir 100\% do

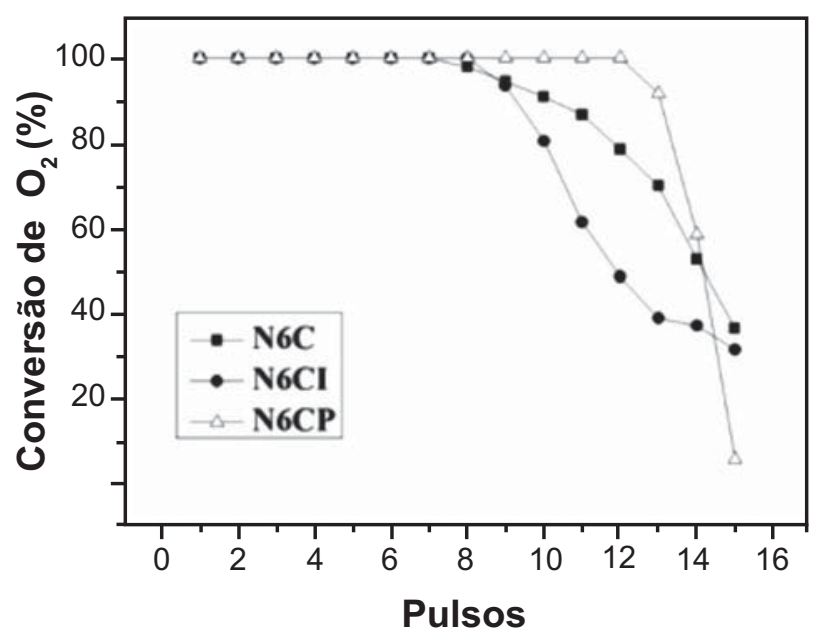

Figura 6: Percentuais de conversão de $\mathrm{O}_{2}$ em função dos pulsos para os carreadores de oxigênio.

[Figure 6: $\mathrm{O}_{2}$ conversion percentage as a function of pulses for the oxygen carrier.]
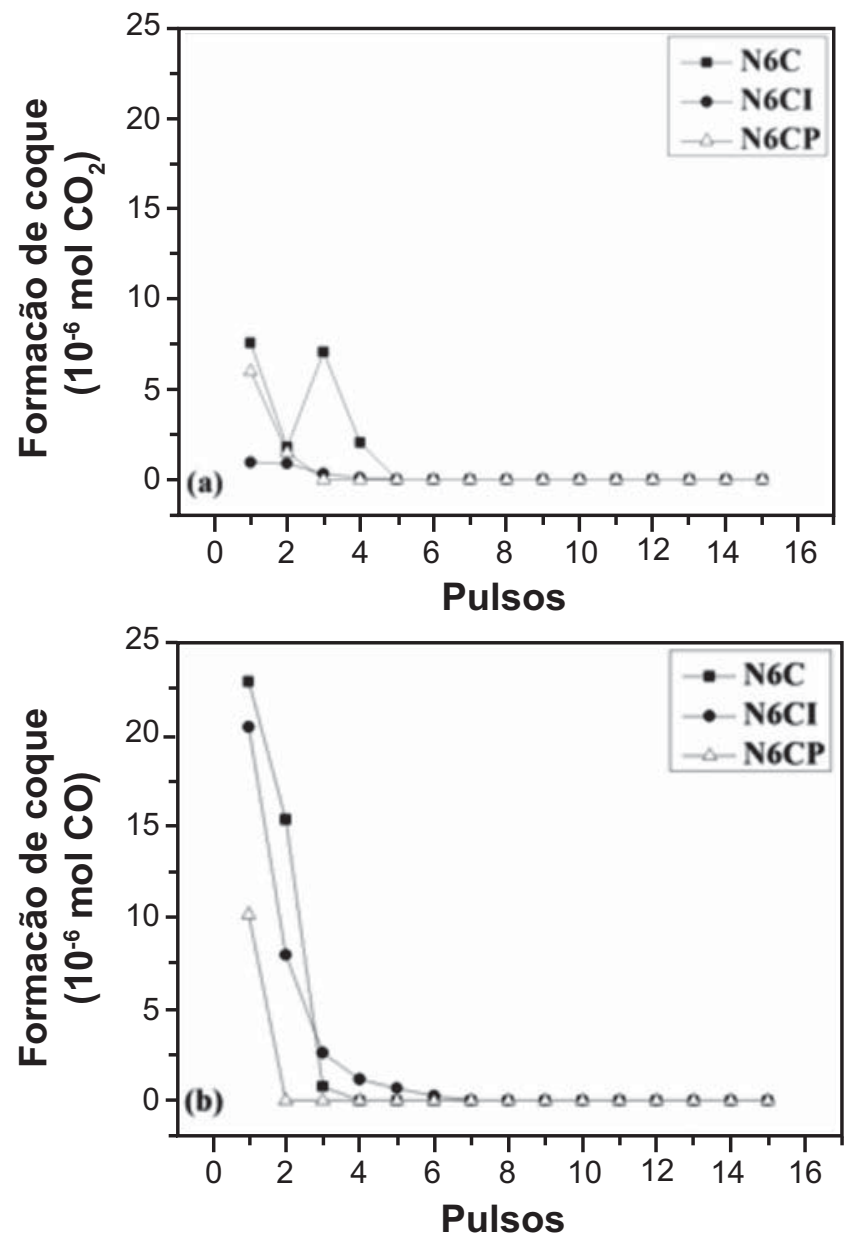

Figura 7: Quantidade de coque em termos de (a) $\mathrm{CO}_{2}$ e (b) $\mathrm{CO}$ dos carreadores.

[Figure 7: Amount of coke in terms of (a) $\mathrm{CO}_{2}$ and (b) $\mathrm{CO}$ of carriers.]

oxigênio até o $12^{\circ}$ pulso. Observa-se uma tendência global em relação à conversão de $\mathrm{O}_{2}$ para os materiais investigados 
da seguinte forma: N6CP $>$ N6C $>$ N6CI.

A Fig. 7 mostra a quantidade de $\mathrm{CO}_{2}$ e $\mathrm{CO}$ formados durante a oxidação, com $\mathrm{O}_{2}$, dos carreadores reduzidos. Pode-se verificar primeiramente a baixa quantidade de coque formada nos materiais, que foi oxidado basicamente até o $3^{\circ}$ pulso. Pode-se inferir pelas Figs. 6 e 7 que primeiramente o carbono é oxidado a $\mathrm{CO}_{2}$ e $\mathrm{CO}$ e somente após a eliminação total do coque é que a fase ativa é oxidada.

O gráfico da Fig. 8 mostra a quantidade de carbono formado por grama de catalisador. Foi verificada a formação de 805,495 e $248 \mu \mathrm{g}$ de carbono por grama de N6C, N6CI e N6CP, respectivamente. Miller e Siriwardane [29] prepararam o carreador de oxigênio $\mathrm{CeO}_{2}-\mathrm{Fe}_{2} \mathrm{O}_{3}$ (com 5\% em massa de $\mathrm{CeO}_{2}$ ), testaram em reações de redução/oxidação utilizando $\mathrm{CH}_{4}$ e $\mathrm{O}_{2}$ e verificaram a formação de $380 \mu \mathrm{g}$ de carbono por grama de carreador na etapa de oxidação. Comparando os resultados obtidos no presente trabalho e aqueles reportados na literatura [29], pode-se perceber que apesar de usar uma quantidade de fase ativa muito maior, o que favorece uma maior formação de carbono, o carreador N6CP apresentou uma quantidade de coque menor. Isso indica que carreadores de oxigênio preparados por coprecipitação tem uma menor tendência à desativação por deposição de carbono em sua superfície. Conforme exposto na Fig. 8, a maior quantidade de carbono depositado por grama de carreador foi apresentada pelo material N6C. Isso pode estar relacionado a não formação de $\mathrm{CO}_{2}$ e $\mathrm{CO}$ nos primeiros quatro pulsos de $\mathrm{CH}_{4}$ (Fig. 4a). A razão $\mathrm{H}_{2} / \mathrm{CO}$ obtida para o N6C e N6CI são consistentes com a reação de craqueamento de carbono (reação mais pronunciada), que produz altas concentrações de carbono e $\mathrm{H}_{2}$, o que corrobora com os dados obtidos na Fig. 5. O restante dos pulsos de $\mathrm{O}_{2}$ consumidos mostrado na Fig. 6 corresponde à oxidação do níquel, segundo a Equação $\mathrm{M}$.

$$
2 \mathrm{Ni}_{(\mathrm{s})}+\mathrm{O}_{2(\mathrm{~g})} \rightarrow 2 \mathrm{NiO}_{(\mathrm{s})}
$$

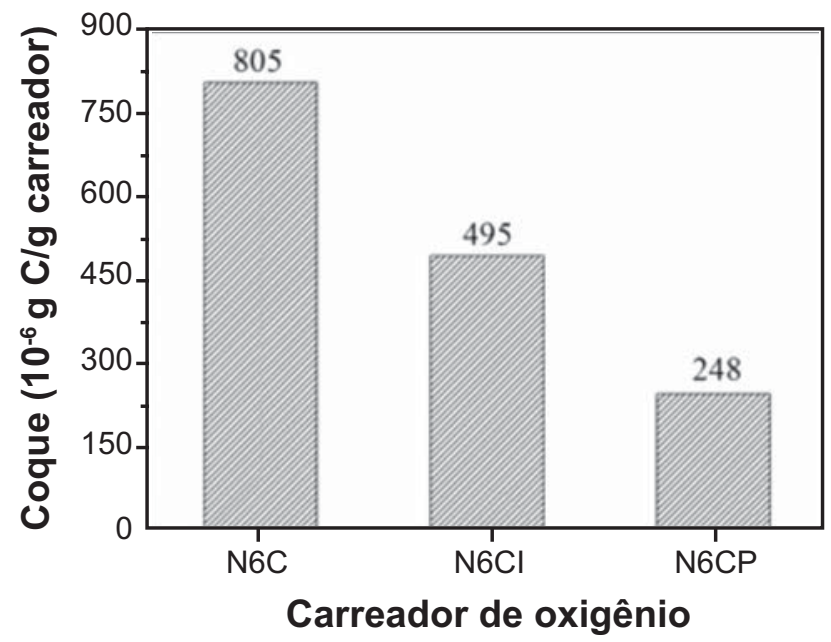

Figura 8: Quantidade de carbono formado por grama de carreador durante a etapa de redução.

[Figure 8: Carbon amount formed per gram of carrier during the reduction step.]

\section{CONCLUSÕES}

Os carreadores de oxigênio N6C, N6CI e N6CP foram sinterizados e testados quanto a sua reatividade para o processo de reforma com recirculação química a temperatura de $900{ }^{\circ} \mathrm{C}$. Os métodos de síntese empregados mostraram-se adequados e exibiram papéis importantes no comportamento dos carreadores de oxigênio obtidos. As fases $\mathrm{LaAlO}_{3}$ e $\mathrm{MgO}\left(\mathrm{La}_{0,995} \mathrm{Al}_{1,05} \mathrm{O}_{3}\right)\left(\mathrm{Al}_{2} \mathrm{O}_{3}\right)_{5}$ obtidas através da síntese por combustão possuem alta estabilidade química e impedem a formação de fases secundárias entre as fases ativas de Ni. Os carreadores apresentaram alto desempenho na conversão de metano para hidrogênio, principalmente através de craqueamento catalítico do $\mathrm{CH}_{4}$, com destaque para o N6C. A amostra N6CP apresentou o melhor desempenho para produção gás de síntese, além de ser apontado como sendo o mais resistente à desativação por coque e com maior potencial de oxidação. Os resultados indicam que o NiO livre na superfície do carreador favorece a rápida desativação, e que o Ni estrutural favorece a reação de craqueamento catalítico do $\mathrm{CH}_{4}$ com consequente deposição de coque. Por fim, os resultados sugerem que o carreador de oxigênio preparado por coprecipitação (N6CP) foi o que apresentou melhor comportamento para a reação de reforma de metano ao longo dos ciclos químicos de oxirredução.

\section{AGRADECIMENTOS}

Ao LabTam e LCR do NUPPRAR, a Universidade Federal do Rio Grande do Norte (UFRN) e a Coordenação de Aperfeiçoamento de Pessoal de Nível Superior (CAPES) pelo apoio financeiro.

\section{REFERÊNCIAS}

[1] IPCC: Climate Change 2007: Synthesis Report, Contribution of Working Groups I, II and III to the Fourth Assessment Report of the Intergovernmental Panel on Climate Change, Eds.: R.K. Pachauri, A. Reisinger, IPCC, Geneva, Switzerland (2007).

[2] O. Levenspiel, Ind. Eng. Chem. Res. 44 (2005) 5073.

[3] J. Adánez, A. Abad, F. Garcia-Labiano, P. Gayan, L.F. De Diego, Prog. Energy Combust. Sci. 38 (2012) 215.

[4] C. Dueso, A. Abad, F. García-Labiano, L.F. De Diego, P. Gayán, J. Adánez, A. Lyngfelt, Fuel 89 (2010) 3399.

[5] S. Wang, G. Lu, J. Millar, Energy Fuels 10 (1996) 896.

[6] M.L.H. Green, S.C. Tsang, J.B. Claridge, Catal. Today 23 (1995) 3.

[7] H. Tian, T. Simonyi, J. Poston, R. Siriwardane, Ind. Eng. Chem. Res. 48 (2009) 8418.

[8] M.R. Quddus, M.M. Hossain, H.I. De Lasa, 1st International Conference on Chemical Looping, Lyon, France (2010).

[9] H.I. Lasa, M.M. Hossain, D. Lopez, J. Herrera, Catal. Today 143 (2009) 179.

[10] H.I. Lasa, M.M. Hossain, K.E. Sedor, Chem. Eng. Sci. 62 (2007) 5464. 
[11] R. Blom, J.E. Readman, A. Olafsen, J.B. Smith, Energy Fuels 20 (2006) 1382.

[12] E. Leal, A.C.F. M. Costa, N.L. Freita, H.L. Lira, R.H.G. A. Kiminami, L. Gama. Mater. Res. Bull. 46 (2011) 1409.

[13] L. Gama, M.A. Ribeiro, B.S. Barros, R.H.A. Kiminami, I.T.Weber, A.C.F.M. Costa. J. Alloy. Compoun. 483 (2009) 453.

[14] S.R. Jain, K.C. Adiga, V.R.P. Verneker, Combust. Flame 40 (1981) 71.

[15] B.S. Barros, Tese Dr., "Reforma a seco e a vapor do metano sobre os precursores catalíticos $\mathrm{LaNiO}_{3} / \alpha-\mathrm{Al}_{2} \mathrm{O}_{3} \mathrm{e}$ $\mathrm{La}_{2} \mathrm{NiO}_{4} / \alpha-\mathrm{Al}_{2} \mathrm{O}_{3}$ preparados por autocombustão assistida por microondas", UFRN, Natal (2009).

[16] R. Rosa, P. Veronesi, C. Leonelli, Chem. Eng. Proc. 71 (2013) 2 .

[17] R. Ciola, Fundamentos da Catálise, Ed. da Universidade de S. Paulo, Editora Moderna, S. Paulo (1981).

[18] M.F. Zawrah, H. Hamaad, S. Meky, Ceram. Int. 33 (2007) 969.

[19] E.N. Alvar, M. Rezaei, H.N, Alvar, Powder Tech. 198 (2010) 275.

[20] J. Berchmans, A.L. Kohl, R.B. And, L.S. Nielsen, A.
Angappana, K.B. Visuvasama, K. Ranjith, Mater. Chem. Phys. 109 (2008) 113.

[21] L.A. Villas-Bôas, D.P.F. De Sousa, Revista Matéria 15, 1 (2010) 001.

[22] M. Parvari, P. Moradi, Iranian J. Chem. Eng. 3, 3 (2006) 29.

[23] M. Johansson, T. Mattisson, A. Lyngfelt, A. Abad, Fuel 87 (2008) 988.

[24] J. Juan, M.C. Román-Martínez, M.J. Illán-Gómez, Appl. Catal. A 355 (2009) 27.

[25] S.S. Lima, H.J. Leea, D.J. Moonb, J.H. Kima, N.C. Parka, J.S. Shina, Y.C. Kima, Chem. Eng. J. 152 (2009) 220. [26] R. Molina, G. Poncelet, J. Catal. 173 (1998) 257.

[27] D.Z. Mezalira, Tese Dr., "Preparação e caracterização de materiais com propriedades catalíticas para a obtenção de hidrogênio e carbono nanoestruturado a partir da decomposição do etanol e do glicerol", UFSC, Florianópolis (2011).

[28] S.M. Stagg-Williams, F.B. Noronha, G. Fendley, D.E. Resasco, J. Catal. 194 (2000) 240.

[29] D.D. Miller, R. Siriwardane, Energy Fuels 27 (2013) 4087.

(Rec. 24/08/2015, Rev. 03/11/2015, Ac. 12/11/2015) 